\title{
Chloroquine Resistant Plasmodium falciparum in Nigeria: Relationship between pfcrt and pfmdr1 Polymorphisms, In-Vitro Resistance and Treatment Outcome
}

\author{
O.A. Folarin ${ }^{1}$, G.O. Gbotosho ${ }^{1}$, A. Sowunmi ${ }^{1}$, O.O. Olorunsogo ${ }^{2}$, A.M.J. Oduola ${ }^{3}$ and T.C. Happi ${ }^{1, *}$ \\ ${ }^{I}$ Malaria Research Laboratories, Institute for Advanced Medical Research and Training (IMRAT), College of Medicine, \\ University of Ibadan, Ibadan, Nigeria; ${ }^{2}$ Department of Biochemistry, University of Ibadan, Ibadan, Nigeria and ${ }^{3}$ Special \\ Program for Research and Training in Tropical Diseases (WHO/TDR), Geneva, Switzerland
}

\begin{abstract}
This study was designed to evaluate the association between polymorphisms in $p f c r t$ and $p f m d r l$ genes and invitro chloroquine (CQ) sensitivity in fresh isolates of $P$. falciparum and patients' treatment outcome. The modified schizont inhibition assay was used to determine in-vitro sensitivity of $P$. falciparum. Polymorphisms in pfcrt and pfmdrl genes were detected using nested PCR and RFLP techniques in 84 P. falciparum isolates obtained from patients with acute uncomplicated malaria.

Eighty five percent (71/84) and 15\% (13/84) of the parasites were resistant and sensitive in-vitro to CQ respectively. Molecular analysis showed presence of mutant pfcrtT76, pfmdr1Y86 and pfmdrlF184 alleles in $60 \%, 33 \%$ and $14 \%$ of the isolates respectively. There was a significant association between in-vitro and in-vivo CQ resistance ( $\mathrm{p}=0.029)$ and also between the presence of mutant $p f c r t 776+p f m d r 1$ Y86-Y184 haplotype and in-vitro ( $\mathrm{p}=0.013)$ or in-vivo CQ resistance $(\mathrm{p}=0.024)$.

Overall results from this study demonstrates that the presence of $p f c r t 776+p f m d r 1$ Y $86-Y 184$ haplotype in Nigerian isolates of Plasmodium falciparum is predictive of in-vitro and in-vivo CQ resistance and therefore may be useful for monitoring resistance to this drug.
\end{abstract}

Key Words: $p f m d r 1, p f c r t$, chloroquine-resistance, in-vitro, in-vivo, Nigeria.

\section{INTRODUCTION}

The emergence and spread of drug resistant malaria parasites in endemic regions has posed a great threat to usefulness of chloroquine (CQ) and sulphadoxine-pyrimethamine (SP), the cheapest and widely used antimalarial drugs. This widespread prevalence of antimalarial drug resistant parasites has led to strong calls for the introduction of artemisinin based combination therapies (ACTs) for treatment of malaria in most endemic areas, as it is expected to represent an effective approach in curbing the development of resistance by Plasmodium falciparum to currently available compounds $[1,2]$. Presently, most malaria endemic countries in Africa including Nigeria have changed their first line antimalarial treatment from $\mathrm{CQ}$ or SP to amodiaquine combined with artesunate or the combination of artemether and lumefantrine. ACTs used in most malaria endemic countries have demonstrated high efficacy, protection against the development of resistance to each component and reduction of malaria transmission [3-5]. However, the relatively high of costs, dosing complexity and the limited experience of their use in sub-Saharan Africa may hamper the widespread deployment of these drug combinations [6]. In addition there

*Address correspondence to this author at the Malaria Research Laboratories, Institute for Advanced Medical Research and Training (IMRAT), College of Medicine, University of Ibadan, Ibadan, Nigeria;

E-mail: christianhappi@ hotmail.com are reports of the emergence of $P$. falciparum with reduced susceptibility to some of the ACTs [7].

This situation therefore points to the fact that other more affordable, effective and readily available combinations are still required. An earlier report [8] has demonstrated the return of CQ susceptible parasites in Malawi soon after the country switched from CQ to SP as the first line therapy for malaria. Furthermore, a recent clinical trial in Malawi also confirmed that CQ had excellent clinical efficacy, 12 years after it was removed from use $[9,10]$. It is therefore necessary to understand the molecular basis of resistance to already available antimalarial drugs such as $\mathrm{CQ}$, and explore the potentials of the information generated in improving the potency and rational for developing effective drug combinations.

The genetic and biochemical basis of CQ resistance in $P$. falciparum has been the subject of several research and has not been fully elucidated in field epidemiologic studies. $P$. falciparum resistance to CQ has been associated with lower drug accumulation in infected erythrocytes [11] or mainly to a mutation at position 76 (K76T) in the Plasmodium falciparum chloroquine resistance transporter ( $p f c r t)$ gene [12, 13]. Several other studies have suggested the implication of multidrug resistance-1 ( $m d r l)$ gene family in resistance to quinoline containing compounds in $P$. falciparum [14-16]. Five point mutations on codons 86, 184, 1034,1042 and 1246 
have been identified to influence the response of $P$. falciparum to CQ $[14,17]$. Several reports have also shown that there is an association between polymorphisms in these two genes and resistance of P. falciparum to CQ in-vitro [12$14,16,18]$, or with CQ therapy failures in field studies [13, 18-26]. While most laboratory reports points to the involvement of these two genes in mediating resistance to CQ and perhaps other drugs, it is yet to demonstrate under field conditions how the allelic differences in pfcrt and pfmdrl genes correlate with the resistant phenotype in different malaria endemic areas of the world, or whether there are differences in resistance mechanisms by parasites in various regions of the world where malaria is endemic.

In this study, the involvement of polymorphisms in pfcrt and pfmdrl genes in in-vitro susceptibility of fresh isolates of $P$. falciparum as well as CQ treatment outcome in children with acute uncomplicated $P$. falciparum malaria in Southwest Nigeria was investigated. Point mutations in $\mathrm{pfcrt}$ and pfmdrl genes were found to be associated with both invitro and in-vivo CQ resistance. The potential implications of these findings in monitoring CQ resistance in areas where ACTs have been introduced for treatment of $P$. falciparum malaria are discussed.

\section{STUDY DESIGN}

\section{Study Site}

The study was carried out at the Malaria Research Laboratory, College of Medicine, University of Ibadan, Nigeria. Malaria in Ibadan is hyperendemic, transmission occurs year round but is more intense from April to October, during the rainy season.

\section{Patients Selection, Treatment and Follow-Up}

Children aged 6months to 12 years with acute symptoms of $P$. falciparum malaria infections were enrolled after clinical examination and microscopic confirmation of infections in a large clinical efficacy study. Informed consent for participation in the study was obtained from parents/guardians of children under the age of 10 years, while assent was obtained from each patient between the ages of 10-12 years. The Joint UI/UCH Institutional Review Committee (IRC) approved the study protocol. Each child was treated with standard dose of CQ $(25 \mathrm{mg} / \mathrm{kg}$ body weight over three days) and followed up for a period of 28 days according to World Health Organization (WHO) protocol [27]. Infection in each child was considered cured if no parasites appeared in the peripheral blood samples during the 28 days follow-up period after treatment. Children in whom parasites reappeared in their blood within the 28 days of follow up were classified as treatment failures and were re-treated with SP to cure the infection.

\section{Determination of In-Vitro Susceptibility of Patient Iso- lates to $\mathbf{C Q}$}

In-vitro susceptibility of each patient isolate of $P$. falciparum to CQ was determined using a modification of the schizont inhibition assay [28]. Briefly, a template containing three-fold serial dilutions of the working CQ solution (9690nM) was prepared in a 96-well microtiter plate. Wells in row $\mathrm{H}$ served as controls without drug. Test plates were derived from each template by transferring $25 \mu 1$ of the drug dilutions to each plate. Two hundred microliters $(200 \mu \mathrm{l})$ of $1 \mathrm{ml}$ parasitized blood diluted in $19 \mathrm{ml}$ of culture medium (RPMI 1640+ HEPES and sodium bicarbonate) was transferred into each well of the plate. Plates containing parasites suspension with CQ in each well were incubated at $37^{\circ} \mathrm{C}$ for 24 to 36 hours in a plexiglass chambers containing a gas mixture $\left(5 \% \mathrm{O}_{2}, 5 \% \mathrm{CO}_{2}, 90 \% \mathrm{~N}_{2}\right)$. The final concentration of CQ in test plate ranged between $969 \mathrm{nM}$ and $1.3 \mathrm{nM}$.

The assay was terminated when at least $60 \%$ of parasites in the control wells (Row H) were schizonts. Each well in a column of 96 well plate was harvested onto glass slides as thick smears, air dried and stained with Giemsa. Parasites development to schizont was determined by counting the number of schizonts against 200 white blood cells in each smear using x100 oil immersion objective of a light microscope. Concentration-response data were analyzed by a nonlinear regression analysis. The 50\% inhibitory concentrations (IC-50) for CQ were calculated using GraphPad Prism version 4.0 for windows software (GraphPad software, San Diego, LA, USA, www.graphpad.com).

According to the WHO criteria [29] of in-vitro CQ sensitivity of $P$. falciparum, a complete inhibition of parasite growth in wells with CQ concentration $<107 \mathrm{nM}(55.5 \mathrm{ng} / \mathrm{ml})$ was considered as sensitive, parasite growth in wells with CQ concentration $>107 \mathrm{nM}$ is defined as resistant and parasite growth in wells of $107 \mathrm{nM} C Q$ concentration but not in wells of higher concentration is defined as borderline sensitivity.

\section{Parasite DNA Extraction}

Parasite genomic DNA was extracted from blood samples collected on filter paper using the chelex extraction method according to the method of Plowe et al. [30].

\section{Analysis of $P$. falciparum Point Mutations in pfcrt and pfmdrl Genes in Isolates Obtained from Patients}

The lysine $(\mathrm{K})$ to threonine $(\mathrm{T})$ mutation at codon 76 of the pfcrt gene was detected by nested PCR followed by RFLP as previously described by Happi et al. [24]. DNA from two laboratory adapted $P$. falciparum clones, 3D7 (Wild type) and Dd2 (mutant) were used as negative and positve controls respectively.

The nested PCR and RFLP methods were also used to evaluate the pfmdr1 N86Y, Y184F S1034C and N1042D point mutations as described previously [26, 31]. The $\mathrm{K} 1$ and 7 G8 laboratory strains of $P$. falciparum presenting with different genotypes at the different codons analyzed were used as controls.

All amplifications were performed in a final volume of 25ul in a PCT- 200 Peltier thermal cycler (MJR Research Inc, MA, USA)

\section{Determination of Plasmodium falciparum Clonal Profile in Infections}

Previous studies [24-26, 32] on molecular analysis of Plasmodium falciparum malaria in Nigerian children following treatment with $\mathrm{CQ}$, have demonstrated that the merozoite surface protein-2 ( $m s p-2)$ was the most informative genetic 
marker to evaluate parasites diversity and the complexity of $P$. falciparum infections in Ibadan, Nigeria. In this study, isolates from each $P$. falciparum infection were characterized on the basis of fragment sizes of alleles of $m s p-2$ after amplification by PCR. Infections were defined as polyclonal if samples from patients showed more than one allele of $F C 27$ or $I C 1 / 3 D 7$ families of $m s p-2$. If an isolate had one allele at each of the families, the clone number was taken to be one. The complexity of infection was calculated as the mean number of distinct fragments of FC27 and IC1/3D7 per PCR-positive sample. Polymorphism in $m s p$ - 2 was also used to distinguish between recrudescence i.e. resistant infection and re-infection. A CQ resistant infection was defined as the occurrence of the same or a subset of the alleles at each of the families (FC27 or IC1/3D7) of $m s p-2$ in the pre and post-treatment samples. A lack of allelic identity in the two families of $m s p$ - 2 in matched pre and post-treatment samples indicated a newly acquired infection.

\section{Data Analysis}

Clinical response to treatment with CQ was expressed as cured (cleared) or resistant (failed). In-vitro profile of $P$. falciparum to CQ was defined as sensitive (S) or resistant (R). Mean values were determined as mean \pm standard deviation (SD). The student $t$-test was used to compare mean values. The Fisher's exact test was used to assess a statistical association between in-vitro susceptibility profiles of patients' isolates of $P$. falciparum to CQ and point mutations in parasites $p f c r t, p f m d r l$ genes and treatment outcome. $P$ value $<0.05$ was considered significant.

\section{RESULTS}

One hundred and twenty children with $P$. falciparum infection were recruited into the study. The mean age of the children was 5.41 \pm 2.94 years (Range 6months-13years). Plasmodium falciparum blood sample was obtained from 84 children by venipuncture for evaluation of the association between in-vitro CQ susceptibility, markers of CQ resistance, and patients treatment outcome. The geometric mean of parasite density in the children at enrollment was 37,451 parasites/ $\mu 1$ of blood (range: 7775 to 150,000 parasites/ $\mu 1$ ). There was no significant difference between the age $(\mathrm{P}=0.82)$, and geometric mean parasites density $(\mathrm{P}=0.60)$ of the 84 children whose blood sample were obtained for invitro $\mathrm{CQ}$ susceptibility and 120 children that were enrolled in the main study.

\section{Response of Infection to Treatment with Standard Regi- men of CQ}

Of the 84 children whose blood samples were obtained for the evaluation of the association between in-vitro susceptibility profile of parasites to CQ, molecular markers of CQ resistance and patients parasitological response to treatment, $62 \%$ (52 of 84 ) and $38 \%$ were classified as cured and treatment failures respectively after PCR correction by $m s p-2$ (Table 1). Mean parasite clearance time (PCT) in the children cured with $\mathrm{CQ}$ was $3.03 \pm 0.41$ days, while the mean fever clearance time (FCT) was $2.12 \pm 0.5$ days after initiation of treatment. Parasites in the group of patients that failed treatment with CQ initially cleared but reappeared between 7 and 28 days (mean recrudescent time $=15.56 \pm 6.03$ days) after commencement of treatment. Infections in 26 out of 32 patients $(81 \%)$ who failed treatment with CQ were classified as RI. Parasites in these patients initially cleared but reappeared between 14 and 28 days after commencement of treatment. RII level of resistance was observed only in six (19\%) of the 32 patients who failed CQ therapy. Parasites in these patients cleared as well but reappeared by day 7 after initiation of treatment.

\section{Parasites Population Structure and Complexity of Infec- tions in Pre- and Post-Treatment Isolates}

Matched sample pairs collected before and after treatment from all 32 patients who failed CQ treatment were successfully analyzed at the $m s p-2$ locus. Alleles were classified according to the size of PCR fragments.

Table 1. Demographic Characteristics of Patients at Enrollment and Patient Treatment Outcome to Chloroquine

\begin{tabular}{|c|c|}
\hline & Treatment with CQ \\
\hline \hline No of patients/isolates & 84 \\
\hline Sex & \\
Male (\%) & $43(49 \%)$ \\
Female (\%) & $41(51 \%)$ \\
\hline Age (years) & \\
Mean \pm SD & $5.41 \pm 2.94$ \\
Range & 6 mths-13years \\
\hline Parasite count (ul $\left.{ }^{-1}\right)$ & \\
Geometric mean & 37,451 \\
Range & $7775-150,000$ \\
\hline In-vivo Treatment outcome & \\
Cured (\%) & $52(62 \%)$ \\
Resistant (\%) & $32(38 \%)$ \\
\hline
\end{tabular}

$\mathrm{SD}=$ Standard deviation.

Genotyping of these samples confirmed our previous report [30] of the presence of different allelic families of $m s p-2$ in parasite DNA derived from a single patient, indicating a polyclonal infection. All pre-treatment isolates were positive for the $m s p-2 I C 1$ and/or FC27 alleles and produced up to eight different fragment sizes (IC1/3D7: 390-1090 bp, FC27: 140-1100 bp). The estimated average number of genetically distinct parasite population as determined with $m s p-2$ in pre- and post-treatment isolates from these 32 patients was 5.3 and 3.8 respectively. There was a significant reduction $(\mathrm{p}=0.03)$ in the number of $m s p-2$ alleles in posttreatment isolates compared to pre-treatment isolates when infections recrudesced.

Detailed analysis of paired pre- and post-treatment isolates from these patients who failed treatment with CQ showed two categories of infection. The first group of infections consisting of $72 \%$ (23 of 32) of patient isolates that failed treatment with $\mathrm{CQ}$, had identical paired PCR fragments at both $F C 27$ and $I C 1$ families of $m s p-2$, indicating genuine recrudescent infections after treatment with $C Q$. The 
second group of infections in the remaining 9 patients showed parasites similar to pre-treatment isolates and the presence of new parasite populations with different genotypes. However, infections in these patients were also considered as treatment failures.

\section{Determination of In-Vitro CQ Susceptibility of Patient Isolates}

Based on the WHO criteria [29] of in-vitro susceptibility of $P$. falciparum to CQ in 84 isolates with successful tests, $15 \%(13 / 84)$ had minimum inhibitory concentration (MIC) of CQ below $107 \mathrm{nM}$ therefore classified sensitive isolates. MIC of CQ $>107 \mathrm{nM}$ was observed in $54 \%(45 / 84)$ of the isolates and classified as resistant isolates. Borderline isolates defined as MIC of $107 \mathrm{nM}$ were observed in $31 \%$ (26/84) of isolates. The mean CQ fifty percent inhibitory concentration (IC-50) for sensitive, borderline and resistant isolates was $6.28 \mathrm{nM}, 13.70 \mathrm{nM}$ and $64.25 \mathrm{nM}$ respectively.

\section{Pfcrt and pfmdr1 Polymorphisms Among Patient Isolates}

Seventy-eight (78), 81 and 70 of the 84 samples were successfully amplified by PCR at locus 76 of pfcrt, loci 86 and 184 of pfmdrl respectively, and were considered for analysis (Table 2 ). Sixty percent $(60 \%)$ of the patients isolates successfully analyzed harbored the mutant pfcrtT76 allele that has been associated with CQ resistance. Ten percent $(10 \%)$ showed a mixed genotype, while $30 \%$ harbored the wild-type pfcrtK76 allele. Analysis of post-treatment samples obtained from the patients who failed CQ treatment showed that isolates obtained from $58 \%$ carried the mutant pfcrtT76 allele. None of the post treatment isolates harbored the wild-type $p f c r t K 76$ allele. Comparison of pre- and posttreatment samples obtained from patients who failed treatment with CQ, showed no increase in the prevalence of mutant pfcrtT76 allele while mixed allele increased from $13 \%$ in pretreatment isolates to $42 \%$ in the post treatment isolates (Table 2).

RFLP analysis of pfmdrl PCR products using AflIII, Dra $I$, Dde $I$ and Ase I restriction enzymes were similarly performed [33] to detect pfmdrl N86Y, Y184F, S1034C and N1042D mutations respectively in isolates obtained from children with $P$. falciparum infection. Mutant pfmdr1Y86 and pfmdrlF184 alleles were present in 33\% (27 of 81) and $14 \%$ (10 of 70$)$ in the pre treatment isolates respectively. All isolates analyzed for S1034C and N1042D mutations harbored the wild type S1034 and N1042 alleles respectively, while $32 \%$ and $82 \%$ of the isolates harbored wild type pfmdr1N86 and pfmdr1Y184 alleles respectively (Table 2). Mixed N86Y and Y184F allele were observed in 35\% and $4 \%$ of the pre treatment isolates respectively. Analysis of post treatment isolates obtained from children who failed CQ treatment showed mutant pfmdrlY86 and pfmdrlF184 alleles in $41 \%$ and $57 \%$ of the isolates respectively. The wild type pfmdr1N86 and pfmdr1Y184 alleles were present in $28 \%$ and $43 \%$ of the post-treatment isolates respectively. None of the post-treatment isolates harbored the mixed allele at codon 184 of pfmdrl gene. However $31 \%$ harbored mixed allele on codon 86 (Table 2 ).

Polymorphisms in pfmdrl gene were clustered into 4 specific haplotypes (haplotypes I- IV). Haplotype I consisted of isolates with both mutant pfmdrlY 86 and pfmdrlF184 alleles (Y86-F184-S1034-N1042). Haplotype II consisted of isolates with wild type pfmdr1N86 and pfmdrlY184 (N86Y184-S1034-N1042), while haplotypes III and IV consisted of isolates with mutant pfmdrlF184 (N86-F184-S1034N1042) and pfmdrlY86 (Y86-Y184-S1034-N1042) respectively (Table 3). In pre-treatment isolates, the pfmdrl haplotype I and II were observed in $7 \%$ and $17 \%$ of the isolates respectively. Haplotypes III and IV were observed in $12 \%$ and $64 \%$ of infections respectively (Table 3). Comparative analysis of pre- and post-treatment isolates showed a significant and strong selection $\left(\mathrm{p}=0.007 ; \chi^{2}=7.13\right)$ of the $p f m d r 1$ haplotype I in post treatment samples obtained from patients who failed CQ treatment (Table 3). Haplotypes II, III and IV were found in $4 \%, 19 \%$ and $46 \%$ of the post treatment isolates respectively (Table $\mathbf{3}$ ).

Table 2. Frequency and Prevalence of pfcrt and pfmdr1 Alleles (At Enrollment) in Peripheral Blood Obtained from Children with Acute Uncomplicated Malaria

\begin{tabular}{|c|c|c|}
\hline Genes and Alleles & Frequency & Prevalence $(\%)$ \\
\hline \multicolumn{3}{|l|}{ K76T Pfcrt $(\mathrm{n}=78)$} \\
\hline $\mathrm{K} 76^{\mathrm{a}}$ & 23 & 30 \\
\hline $\mathrm{T} 76^{\mathrm{b}}$ & 47 & 60 \\
\hline $\mathrm{K} 76+\mathrm{T} 76$ & 8 & 10 \\
\hline \multicolumn{3}{|l|}{$\mathrm{N} 86 \mathrm{Y}$ pfmdrl $(\mathrm{n}=81)$} \\
\hline $\mathrm{N} 86^{\mathrm{a}}$ & 26 & 32 \\
\hline Y $86^{\mathrm{b}}$ & 27 & 33 \\
\hline $\mathrm{N} 86+\mathrm{Y} 86$ & 28 & 35 \\
\hline \multicolumn{3}{|l|}{$\mathrm{Y} 184 \mathrm{~F} p f m d r 1(\mathrm{n}=70)$} \\
\hline $\mathrm{Y} 184^{\mathrm{a}}$ & 57 & 82 \\
\hline F184 & 10 & 14 \\
\hline $\mathrm{Y} 184+\mathrm{F} 184$ & 3 & 4 \\
\hline
\end{tabular}

Pfcrt: P. falciparum CQ resistance transporter and pfmdrl: P. falciparum multiple drug resistance 1 gene

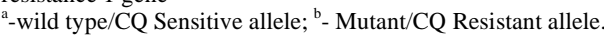

Assessment of the combination of pfcrt alleles at codon 76 with $p f m d r 1$ haplotypes in pre and post-treatment samples obtained from patients showed that the wild type pfcrtK76 allele+any pfmdrl haplotypes was only present in $27 \%$ $(22 / 82)$ of the pre treatment isolates. Mutant pfcrtT76+ pfmdr1 haplotype I (Y86-F184-S1034-N1042) was present in $2 \%(2 / 82)$ and $31 \%(8 / 26)$ of pre and post-treatment isolates respectively. Interestingly, the mutant pfcrt 776 allele combined with pfmdrl haplotype IV (Y86-Y184-S1034$\mathrm{N} 1042$ ), was observed in $50 \%$ and $46 \%$ of all pre- and posttreatment isolates respectively. None of the post treatment isolates obtained from patients that failed CQ treatment harboured the wild type $p f c r t K 76$ allele+any $p f m d r 1$ haplotype (Table 4).

\section{Association between In-Vitro Chloroquine Susceptibility and Gene Polymorphisms}

The in-vitro sensitivity profile according to WHO criteria showed that 45 out of 84 isolates were resistant to CQ. Correlation analysis between CQ in-vitro susceptibility and gene polymorphisms showed that there was a significant associa- 
Table 3. Mutations Patterns and Prevalence in pfmdr1 Gene in Isolates of $P$. falciparum Obtained from Children with Uncomplicated Malaria at Enrollment and after Treatment with CQ

\begin{tabular}{|c|c|c|c|c|c|c|c|}
\hline \multicolumn{9}{|c|}{ PFMDRICODONS } \\
\hline Haplotypes & $\mathbf{8 6}$ & $\mathbf{1 8 4}$ & $\mathbf{1 0 3 4}$ & $\mathbf{1 0 4 2}$ & $\begin{array}{c}\text { Pre-Treatment (\%) } \\
{[\mathbf{n}=\mathbf{7 0 ]}}\end{array}$ & $\begin{array}{c}\text { Post-Treatment (\%) } \\
{[\mathbf{n}=\mathbf{2 6}]}\end{array}$ \\
\hline \hline I & Tyr (Y) & Phe (F) & Ser (S) & Asn (N) & $5(7 \%)$ & $8(31 \%)$ & $0.007 *(7.13)$ \\
\hline II & Asn & Tyr & Ser & Asn & $12(17 \%)$ & $1(4 \%)$ & 0.17 \\
\hline III & Asn & Phe & Ser & Asn & $8(12 \%)$ & $5(19 \%)$ & $12(46 \%)$ \\
\hline IV & Tyr & Tyr & Ser & Asn & $45(64 \%)$ & 0.5 \\
\hline
\end{tabular}

Mutant allele in boldface; $\operatorname{Tyr}(\mathrm{Y})=$ Tyrosine; Asn $(\mathrm{N})=$ Asparagine; Phe $(\mathrm{F})=$ Phenylalanine; Ser $(\mathrm{S})=$ Serine; * $\mathrm{P}$ value statistically significant.

Table 4. Prevalence of $p$ fcrt and pfmdrl Polymorphisms in Pre-Treatment and Post-Treatment Isolates Obtained from Children Infected with $P$. falciparum

\begin{tabular}{|c|c|c|}
\hline Alleles of Genes and Haplotype & Pre Treatment (\%) n=82 & Post Treatment (\%) n=26 \\
\hline \hline$P f c r t$ K76+any $p f m d r l$ haplotype & $22(27)$ & $(0)$ \\
\hline$P f c r t T 76+p f m d r l$ haplotype I & $2(2)$ & $1(31)$ \\
\hline$P f c r t T 76+p f m d r l$ haplotype II & $12(15)$ & $5(19)$ \\
\hline$P f c r t T 76+p f m d r l$ haplotype III & $5(6)$ & $12(46)$ \\
\hline$P f c r t T 76+p f m d r l$ haplotype IV & $41(50)$ & \\
\hline
\end{tabular}

$\mathrm{n}=$ Number of isolates.

tion between in-vitro CQ resistance and mutant pfcrtT76 $(\mathrm{p}=0.013 ; \mathrm{OR}=3.553 ; 95 \% \mathrm{CI}=1.278-9.875)$, wild-type $\mathrm{pfmdr}$ $1 Y 184(\mathrm{p}=0.029 ; \mathrm{OR}=0.203 ; 95 \% \mathrm{CI}=0.05-0.818)$, pfmdrl Y86-Y184-S1034-N1042 haplotype $(\mathrm{p}=0.004 ; \mathrm{OR}=4.133$; $95 \% \mathrm{CI}=1.555-10.99)$, mutant $p f c r t \mathrm{~T} 76+p f m d r 1 Y 86(\mathrm{p}=0.028$; $\mathrm{OR}=2.706 ; 95 \% \mathrm{CI}=1.103-6.635)$, mutant pfcrtT76+ pfmdrl Y86-Y184-S1034-N1042 $\quad(\mathrm{p}=0.046 ; \quad \mathrm{OR}=2.464 ; 95 \% \mathrm{CI}=$ 1.009-6.002). There was a strong association $(\mathrm{p}=0.011$; $\mathrm{OR}=0.27 ; 95 \% \mathrm{CI}=0.096-0.764$ ) between the presence of the pfcrtK76 allele and CQ sensitivity despite the presence of any pfmdrl haplotype (Table 5).

\section{Association between Gene Polymorphisms and Patient Treatment Outcome}

Univariate analyses showed that there was a significant association between CQ treatment failure and the presence of pfmdrlY86-Y184 haplotype $(\mathrm{p}=0.022 ; \mathrm{OR}=3.36 ; 95 \% \mathrm{CI}=$ 1.157-9.759) or mutant pfcrtT76+pfmdr1Y86-Y184 haplotype $(\mathrm{p}=0.024 ; \mathrm{OR}=2.864 ; 95 \% \mathrm{CI}=1.138-7.209)($ Table 6).

\section{Association between In-Vitro CQ Susceptibility and In- Vivo Treatment Outcome}

The correlation between in-vitro susceptibility profiles of $P$. falciparum isolates obtained from patients and responses of infection to CQ showed that among the 32 patients who failed CQ treatment, 22 were resistant to CQ in-vitro, while $10(31 \%)$ had parasites that were sensitive to the drug. $P$. falciparum isolates obtained from 23 (44\%) of the 52 patients who were cured with CQ, showed a resistant profile to $\mathrm{CQ}$ in-vitro. A significant association ( $\mathrm{p}=0.029$; $\mathrm{RR}=2.774$; $95 \% \mathrm{CI}=1.098-7.005)$ was observed between in-vivo treatment outcome and in-vitro sensitivity of $P$. falciparum isolates to $\mathrm{CQ}$.

\section{DISCUSSION}

This study has demonstrated high level P.falciparum resistance to $\mathrm{CQ}$ in-vitro and in-vivo in Ibadan, Southwest Nigeria. In-vitro and in-vivo CQ resistances were $54 \%$ and $38 \%$ respectively. The current level of in-vivo resistance (38\%) is apparently lower compared to 51\% [24-26] reported previously from the same study site. This sharp reduction in the level of in vivo CQ resistance may be explained by the 2005 antimalarial treatment policy change from CQ to artemether-lumefantrine or artesunate-amodiaquine as first lines treatments for acute uncomplicated malaria in Nigeria, although, chloroquine has not been completely withdrawn, despite the change in drug policy. It has been argued that the withdrawal of CQ or SP as first line treatment for uncomplicated malaria in other disease endemic settings of Africa has led to the reemergence of drug sensitive Plasmodium falciparum [8-10]. The fact that in-vitro CQ resistance $(54 \%)$ is higher than in-vivo resistance $(38 \%)$, may be attributed to immunopotentiation of antimalarial drugs by patients immunity, which would have helped older patients (>5years of age) clear drug CQ resistant parasites. 
Table 5. Association between $p f c r t / p f m d r 1$ Mutations in P. falciparum Isolates Obtained from Children at Enrollment and CQ InVitro Susceptibility Profile

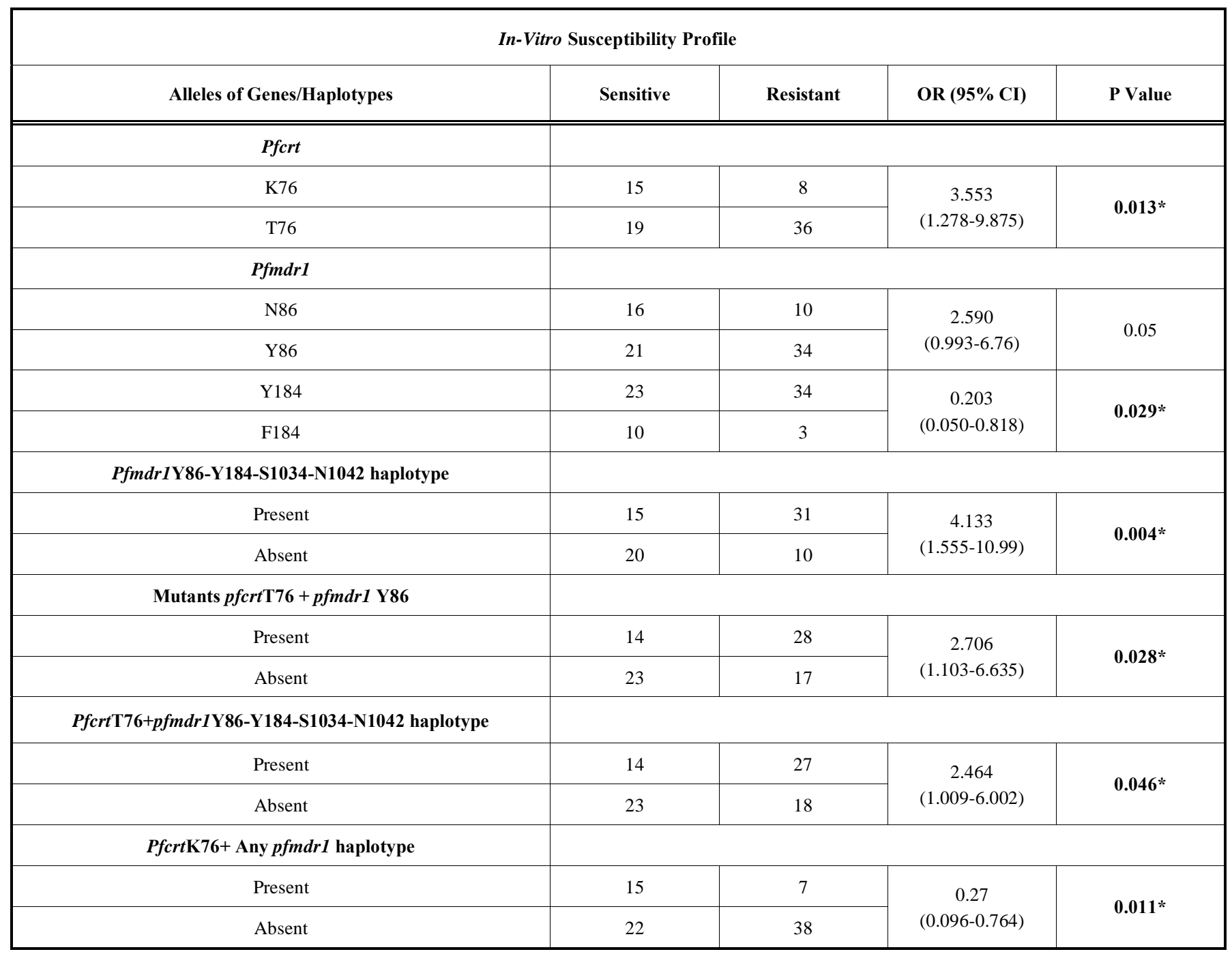

*P value statistically significant; Alleles of $p f c r t$ and $p f m d r l$ genes are the same as described in Table 2.

Previous reports from malaria endemic areas have shown that children over the age of 5 years who have acquired some level of immunity to Plasmodium falciparum can clear drug resistant parasites [26, 32-36]. The value of in-vitro susceptibility testing of $P$. falciparum for elucidating the epidemiology of drug resistant malaria cannot be overemphasized. This study showed a strong association $(\mathrm{p}=0.029)$ between resistance to CQ in-vitro and in-vivo, confirming the fact that despite the technical challenges posed by the in-vitro technique in disease endemic countries of Africa, it is still a very useful tool for monitoring drug resistance. To our knowledge, this study is one of the very few studies in West Africa that shows a correlation between Plasmodium falciparum resistance to CQ in-vitro and in-vivo resistance to the drug.

This study showed similarities in the parasite msp-2 genotype from pre and post treatment infections in $72 \%(23$ of 32) of the children who failed CQ treatment. The rest of the isolates (9) obtained from children that failed CQ treatment showed the presence of new infections in the post treatment isolates in addition to similarities in the pre and post treatment infections. These new infections observed in the post treatment isolates are confirming the polyclonality of infections observed in this study and in previous study in the same area $[24,26,32]$. It is possible that these new genotypes represent minor parasite populations in pre-treatment isolates amplified by PCR below the threshold of ethidium bromide detection. Polyclonality of infections may also have implications in epidemiology of antimalarial drug resistance. Ten patients who failed CQ treatment in spite of the parasite sensitivity to CQ in-vitro is one of the discrepancies between in-vitro and clinical outcome. One possible reason to this may be the complexity of infection as shown by msp- 2 genotyping. It is possible that pre-treatment isolates consisted of parasites with different drug susceptibilities. In this case, a major population sensitive to CQ would have been cleared by the drug leaving a minor population which is resistant to CQ.

This may be supported by the fact that 6 of these isolates have borderline in-vitro sensitivity to $\mathrm{CQ}$ and, from the msp2 genotyping, a significant $(\mathrm{p}=0.03)$ reduction was observed 
Table 6. Association between pfcrt/pfmdr1 Mutations in P. falciparum Isolates Collected from Children at Enrollment and CQ Treatment Outcome

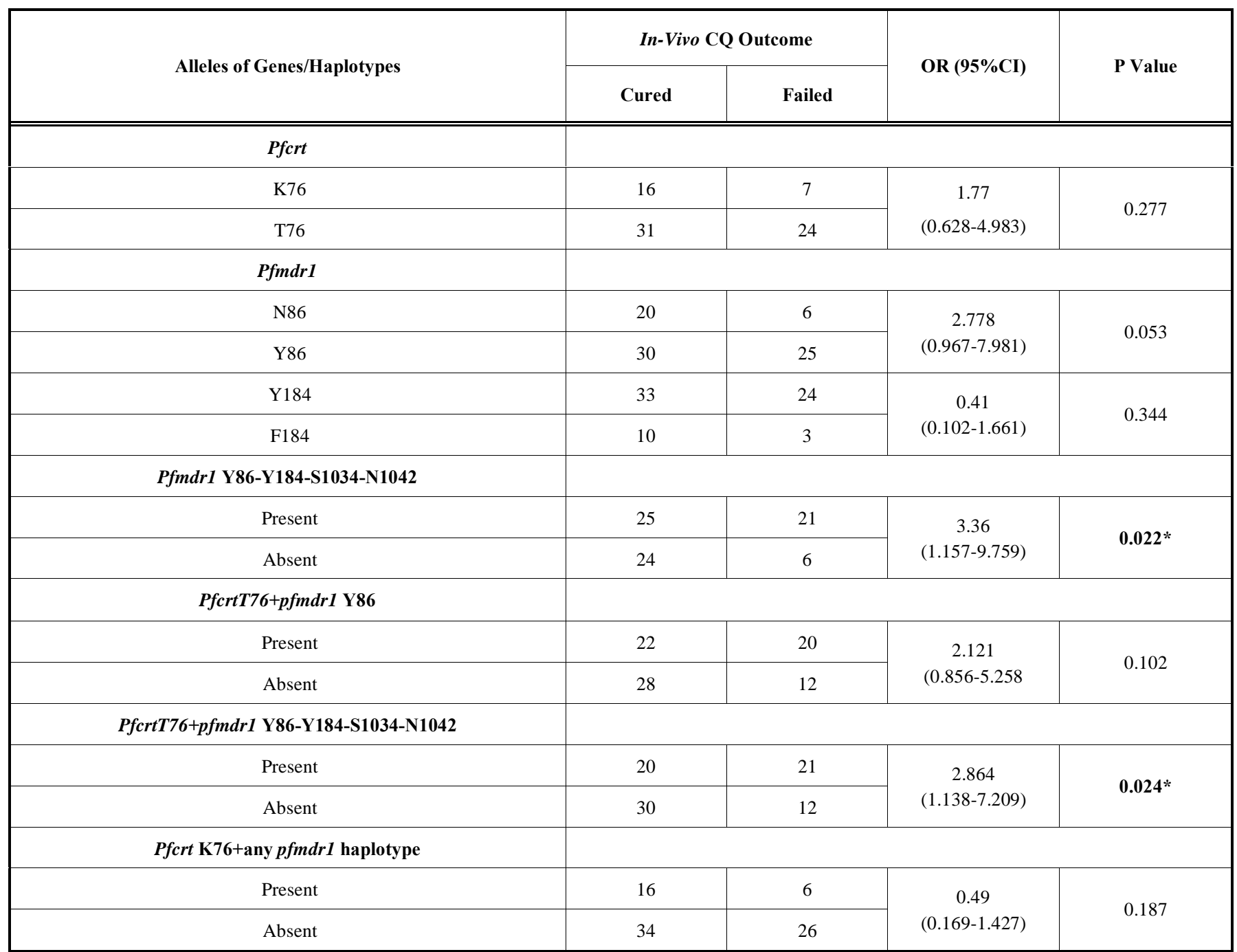

*P value statistically significant.

Alleles of pfcrt and pfmdrl genes are the same as described in Table 2.

in the average number of clones (3.8) in the post treatment isolates compared to pre treatment isolates (5.3).

Although point mutations in pfcrt and pfmdrl genes as molecular markers of $\mathrm{CQ}$ resistance represent valuable tools for surveillance and monitoring changes in CQ efficacy, the interplay between polymorphisms in these two genes is not fully understood, as they may account for the differences observed in different malaria epidemiological settings [37]. This study analyzed the association of mutations at codon 76 of pfcrt gene and codons 86, 184, 1034 and 1042 of pfmdr1 gene with in-vitro and in-vivo CQ resistant or sensitive phenotypes. The pfmdrlY184F mutation was identified in $14 \%$ (10 of 70) of isolates analyzed in this study. This point mutation has been reported to be associated with higher in-vitro resistance in laboratory strains [14] and field isolates from South America [38, 39]. No isolate analyzed in this study showed mutants PfmdrlC1034 or pfmdr1D1042. This is in contrast with previous observations made in South America where these two alleles have been associated with high grade CQ resistance in isolates of $P$. falciparum $[39,40]$.
Correlation analysis between mutations in pfcrt and pfmdrl genes and in-vitro CQ susceptibility in 82 isolates for which both in-vitro and molecular data were available, showed a very interesting feature. Some isolates of $P$. falciparum harboring the mutant $p f c r t T 76$ and pfmdrl Y86 alleles were sensitive to $\mathrm{CQ}$ in-vitro. These finding are similar to earlier reports from Senegal [41], the Philippines [42] and South East Asia [24, 43].

The occurrence of molecular markers of CQ resistance (mutant pfcrtT76 or pfmdrl Y86 alleles) in some isolates of $P$. falciparum that are CQ sensitive in-vitro can be attributed to either the involvement of compensating mutations in either these two genes or other P. falciparum genes not previously associated with modification of drug response.

In addition, a recent report from Johnson and colleagues [43] showed that the presence of a mutation at codon 163 of the pfcrt (S163R) gene, where a serine (S) is replaced by an arginine ( $\mathrm{R})$ is associated with CQ sensitivity in some isolates of Plasmodium falciparum, despite the presence of the 
pfcrtT76 mutation. However, the role of parasites population dynamics on the detection of either the mutant or wild-type alleles of both pfcrt and pfmdrl depends on the predominant parasites populations in patients' isolates and cannot be ignored in an area of intense transmission like Ibadan, Nigeria. Further studies are needed for a better understanding of the association between in-vitro susceptibility of parasites to $\mathrm{CQ}$, markers of $\mathrm{CQ}$ resistance and clinical outcome in this area of high malaria transmission.

Although there was no association between in-vitro CQ resistance and mutant $p f m d r 1 \mathrm{Y} 86$ alone, association between the double mutant pfcrtT76+pfmdrlY86 or pfmdrlY86Y184 haplotype confirms the involvement of $p f m d r 1$ in CQ resistance in-vitro. This is in agreement with previous observations in South East Asia where pfmdrl Y86-Y184-S1034N1042 haplotype is associated with CQ resistance in cultured isolate [44]. Previous reports [21, 24, 26, 45] have shown the primary role of the mutant pfcrt $\mathrm{T} 76$ as the major determinant of CQ resistance while mutation in $p f m d r l$ gene plays a modulatory role in the mechanism of resistance. It is also possible that the presence of wild type $p f m d r Y 184$ in isolates harboring mutant $p f m d r 1 Y 86$ also confer more fitness on the parasite against CQ.

The association observed between mutant pfcrtT76 allele+pfmdr1 Y86-Y184 haplotype and in-vitro CQ resistance in our study further confirms the role of polymorphisms in these two genes in the mechanism of CQ resistance in $P$. falciparum. This is further strengthened by one interesting observation in our study, as we show an association $(\mathrm{p}=0.011)$ between in-vitro CQ sensitivity and the presence of wild type pfcrtK76 allele+ any pfmdrl haplotype (Table 5). This observation demonstrates and confirms the primary and important role of pfcrt polymorphisms, irrespective of pfmdrl polymorphisms and perhaps other genes in P. falciparum CQ resistance.

It has been difficult to establish an association between the Plasmodium falciparum mutations and patients' treatment outcomes in Ibadan, Nigeria [24] and in many other malaria endemic countries of Africa and Madagascar [37]. The high prevalence $(60 \%)$ of the mutant pfcrtT76 allele in pre-treatment isolates of all patients treated with CQ in this study makes it difficult for this allele to be predictive of treatment failures. This high prevalence of the pfcrtT76 allele is consistent with rates ranging from $60 \%$ to $100 \%$ reported in other malaria endemic regions $[13,22,23,33,41$, 46-48].

Unlike the observations made in-vitro, a significant association was observed between CQ treatment failure and pfmdr1 Y86-Y184 haplotype $(\mathrm{p}=0.022)$ or mutant pfcrt $\mathrm{T} 76+$ pfmdr1 Y86-Y184 haplotype $(\mathrm{p}=0.024)$ (Table 6). The significant association between in-vivo CQ failure and pfmdrl Y86-Y184 haplotype or mutant pfcrtT76+pfmdr1Y86-Y184 confirms that polymorphisms on these two genes are involved in in-vivo CQ resistance. The mutant pfmdr1 Y86F184 haplotype was observed to significantly $(p=0.007)$ increase in post treatment isolates compared to pre treatment samples indicating a selection of the haplotype. The reasons for this selection remain unclear.

Some patients treated with standard doses of CQ cleared infections containing mutant allele of pfcrt and/or pfmdrl that have been associated with both in-vitro and in-vivo CQ resistance. The ability of such patients to clear parasites with mutant genotype may be due to acquired immunity, as previously shown in other studies $[26,32,35]$.

\section{CONCLUSIONS}

Overall, this study showed that the mutant pfcrt $776+$ pfmdr1Y86-Y184 haplotype is associated with in-vitro and in-vivo CQ resistance and can be used to identify Plasmodium falciprum resistant phenotypes in isolates from Nigeria. To our knowledge, this is one of the few studies in West Africa that clearly demonstrates the role of parasites mutations in phenotypic CQ resistance in-vitro and in-vivo. Further studies in other malaria endemic countries are needed in order to validate these findings, especially, in areas where ACTs have been introduced, and there is a need to monitor the return of CQ sensitivity.

\section{ACKNOWLEDGEMENTS}

The authors thank all the patients, their parents or guardians for volunteering to participate in the study. We thank MR4 for providing all genomic DNA used as controls for PCR and RFLP experiments.

This study was supported by International Atomic Energy Agency (IAEA) project RAF/0625, the Harvard Malaria Initiative, the NIH/Fogarty International Centre and the Multilateral Initiative for Malaria in Africa (MIM)/TDR project ID A20239. Onikepe A Folarin was supported by a Postgraduate student fellowship of the Federal Government of Nigeria. Christian T Happi is supported by a Fogarty International Research Collaboration Award (FIRCA) no. NIH RO3TW007757 and a UNICEF/UNDP/World Bank/WHO/ TDR Grant ID A50337.

\section{REFERENCES}

[1] White NJ. Antimalaria drug resistance and combination chemotherapy. Philosophical Transactions of the Royal Society of London. Series B: Biol Sci 1999; 354: 739-49.

[2] White NJ. Delaying antimalarial resistance with combination therapy. Parasitologia 1999; 41: 301-8.

[3] White NJ. Preventing antimalarial drug resistance through combinations. Drug Resist Update 1998; 1(1): 3-9.

[4] Bloland PB, Ettling M, Meek S. Combination therapy for malaria in Africa: hype or hope? Bull World Health Organ 2000; 78(12): 1378-88

[5] Sutherland CJ, Ord R, Dunyo S, et al. Reduction of malaria transmission to Anopheles mosquitoes with a six-dose regimen of coartemether. PLoS Med 2005; 2(4): e92.

[6] Bloland PB, Kachur SP, Williams HA. Trends in antimalarial drug deployment in sub- Saharan Africa. J Exp Biol 2003; 206(21): 3761-9.

[7] Jambou R, Legrand E, Niang M, et al. Resistnce of Plasmodium falciparum field isolates to in-vitro artemether and point mutations of the SERCA type pfATPase 6. Lancet 2005; 366(9501): 1960-3.

[8] Kublin JG, Cortese JF, Njunju EM, et al. Reemergence of chloroquine-sensitive Plasmodium falciparum malaria after cessation of chloroquine use in Malawi. J Infect Dis 2003; 187: 1870-5.

[9] Laufer MK, Thesing PC, Eddington ND, et al. Return of chloroquine antimalarial efficacy in Malawi. N Engl J Med 2006; 355 : $1959-66$.

[10] Laufer MK, Djimde AA, Plowe CV. Monitoring and deterring drug resistant malaria with era of combination therapy. Am J Trop Med Hyg 2007; 77(6): 160-9.

[11] Krogstand DJ, Gluzman IV, Kyle DE, et al. Efflux of chloroquine from Plasmodium falciparum: Mechanism of chloroquine resistance. Science 1987; 238: 1283-5. 
[12] Fidock DA, Nomura T, Talley AK, et al. Mutations in the P. falciparum digestive vacuole transmembrane protein $P f_{c r t}$ and evidence for their role in chloroquine resistance. Mol Cell 2000; 6: 861-71.

[13] Djimde A, Doumbo OK, Cortese JF, et al. A molecular marker for chloroquine-resistant falciparum malaria. N Engl J Med 2001; 344: 257-63.

[14] Foote SJ, Kyle DE, Martin RK, et al. Several alleles of the multidrug-resistance gene are closely linked to chloroquine resistance in Plasmodium falciparum. Nat 1990; 345: 255-8.

[15] Duraisingh MT, Drakeley CJ, Muller O, et al. Evidence for selection for the tyrosine-86 allele of the $p f m d r 1$ gene of Plasmodium falciparum by chloroquine and amodiaquine. Parasitology 1997; 114: 205-11.

[16] Reed MB, Saliba, KJ, Caruana SR, Kirk K, Cowman AF. Pgh1 modulates sensitivity and resistance to multiple antimalarials in Plasmodium falciparum. Nature 2000; 403: 906-9.

[17] Wellems TE, Panton LJ, Gluzman LY, et al. Chloroquine resistance not linked to mdr-like genes in a Plasmodium falciparum cross. Nat Lond 1990; 345: 253-5

[18] Adagu IS, Dias F, Pinheiro L, Rombo L, do Rosario V, Warhurst DC. Guinea Bissau: association of chloroquine resistance of Plasmodium falciparum with the Tyr86 allele of the multiple drugresistance gene Pfmdr1. Trans R Soc Trop Med Hyg 1996; 90: 901 .

[19] Basco LK, Le Bras J, Rhoades Z, Wilson CM. Analysis of pfmdr1 and drug susceptibility in fresh isolates of Plasmodium falciparum from sub-Saharan Africa. Mol Biochem Parasitol 1995; 74: 157-66.

[20] Basco LK, de Pecoulas PE, Le Bras J, Wilson CM. Plasmodium falciparum: molecular characterization of multidrug- resistant Cambodian isolates. Exp Parasitol 1996; 82: 97-103.

[21] Dorsey G, Kamya MR, Singh A, Rosenthal PJ. Polymorphisms in the Plasmodium falciparum pfcrt and pfmdr-1 genes and clinical response to chloroquine in Kampala, Uganda. J Infect Dis 2001; 183: $1417-20$.

[22] Durand R, Jafari S, Vauzelle J, Delabre JF, Jesic Z, Le Bras J. Analysis of pfcrt point mutations and chloroquine susceptibility in isolates of Plasmodium falciparum. Mol Biochem Parasitol 2001; 114: 95-102.

[23] Omar SA, Adagu IS, Gump DW, Ndaru NP, Warhurst DC. Plasmodium falciparum in Kenya: high prevalence of drug-resistanceassociated polymorphisms in hospital admissions with severe malaria in an epidemic area. Ann Trop Med Parasitol 2001; 95: 661-9.

[24] Happi TC, Thomas SM, Gbotosho GO, et al. Point mutations in the pfcrt and pfmdr-1 genes of Plasmodium falciparum and clinical response to chloroquine, among malaria patients from Nigeria. Ann Trop Med Parasitol 2003; 97(5): 439-51.

[25] Happi TC, Gbotosho GO, Sowunmi A, et al. Molecular analysis of recrudescent Plasmodium falciparum malaria infections in children treated with chloroquine in Nigeria. Am J Trop Med Hyg 2004; 70 (1): 20-6.

[26] Happi CT, Gbotosho, GO, Folarin, OA, et al. Linkage disequilibrium between two distinct loci in chromosomes 5 and 7 of Plasmodium falciparum and in-vivo chloroquine resistance in Southwest Nigeria. Parasitol Res 2006; 100: 141-48.

[27] WHO: Assessment of therapeutic efficacy of antimalarial drugs for uncomplicated malaria in areas with intense transmission. WHO/MAL1996/96-1077, World Health Organization, Geneva 1996.

[28] Oduola AM, Omitowoju GO, Gerena L, et al. Reversal of mefloquine resistance with penfluridol in isolates of Plasmodium falciparum from south-west Nigeria. Trans R Soc Trop Med Hyg 1993; 87: 81-3

[29] WHO: In vitro micro-test for the assessment of the response of Plasmodium falciparum to chloroquine, mefloquine, quinine, sulfadoxine/pyrimethamine and amodiaquine. WHO/MAP/87.2. Technical Report 1990; World Health Organization, Geneva 1990.

[30] Plowe CV, Djimde AA, Bouare M, Doumbo O, Wellems TE. Pyrimethamine and proguanil resistance-conferring mutations in Plamodium falciparum dihydrofolate reductase: Polymerase chain reaction methods for surveillance in Africa. Am J Trop Med Hyg 1995; 52: 565-8.

[31] Duraisingh MT, Jones P, Sambou I, Von Seidlein L, Pinder M, Warhurst DC. The tyrosine-86 allele of the pfmdrl gene of Plasmodium falciparum is associated with increased sensitivity to the anti-malarials mefloquine and artemisinin. Mol Biochem Parasitol 2000; 108(1): 13-23.

[32] Happi CT, Gbotosho GO, Folarin OA, et al. Polymorphisms in Plasmodium falciparum dhfr and dhps genes and age related invivo sulfadoxine-pyrimethamine resistance in malaria-infected patients from Nigeria. Acta Tropica 2005; 95: 183-93.

[33] Pati SS, Mishra S, Mohanty S, et al. Pfcrt haplotypes and in-vivo chloroquine response in Sunderarh district, Orissa, India. Trans R soc Trop Med Hyg 2007; 101(7): 650-4.

[34] White NJ. The assessment of antimalarial drug efficacy. Trends in parasitol 2002; 18: 458-64.

[35] Djimde AA, Doumbo OK, Traore O, et al. Clearance of drugresistant parasites as a model for protective immunity in Plasmodium falciparum malaria. Am J Trop Med Hyg 2003; 69(5): 55863.

[36] Casey GJ, Ginny M, Uranoli M, et al. Molecular analysis of Plasmodium falciparum from drug treatment failure patients in Papua New Guinea. Am J Trop Med Hyg 2004; 70: 251-55.

[37] Rason MA, Andrianantenaina HB, Ariey F, Raveloson A, Domarle O, Randrianarivelojosia M. Prevalent pfmdr1 n86y mutant Plasmodium falciparum in Madagascar despite absence of pfcrt mutant strains. Am J Trop Med Hyg 76 (6): 1079-83.

[38] Vieira PP, Ferreira MU, Alecrim MG, et al. Zalis. Pfcrt polymorphism and the spread of chloroquine resistance in Plasmodium falciparum populations across the Amazon basin. J Infec Dis 2004; 190(2): 417-24.

[39] Zalis MG, Pang L, Silveira MS, Milhous WK, Wirth D. Characterization of Plasmodium falciparum isolated from the Amazon region of Brazil: evidence for quinine resistance. Am J of Trop Med Hyg 1998; 58: 630-7.

[40] Huaman MC, Roncalm Nakazawa S, Ailong T, et al. Polymorphisms of the Plasmodium falciparum multidrug resistance and CQ resistance transporter genes and In-vitro susceptibility to aminoquinolines in isolates from the Peruvian Amazon. Am J Trop Med Hyg 2003; 70(5): 461-6.

[41] Thomas SM, Ndir O, Dieng T, et al. In-vitro chloroquine susceptibility and PCR analysis of pfcrt and pfmdrl polymorphisms in Plasmodium falciparum isolates from Senegal. Am J Trop Med Hyg 2002; 66: 474-80.

[42] Chen N, Kyle DE, Pasay C, et al. Pfcrt alleleic types with two novel amino acid mutations in chloroquine-resistant Plasmodium falciparum isolates from the Philippines. Antimicrob Agents Chemother 2003; 47 (11): 3500-5.

[43] Johnson DJ, Fidock DA, Mungthin M, et al. Evidence for the central role of pfcrt in conferring Plasmodium falciparum resistance to diverse antimalarial agents. Mole Cell 2004; 15: 867-77.

[44] Pickard AL, Wongsrichanalai C, Purfield A, et al. Resistance to antimalarials in South east Asia and genetic polymorphisms in pfmdr1. Antimicrob Agent Chemother 2003; 47 (8): 2418-23.

[45] Pillai DR, Labbe AC, Vanisaveth V, et al. Plasmodium falciparum malaria in Laos, chloroquine treatment outcome and predictive value of molecular markers. J Infec Dis 2001; 183: 789-95.

[46] Mlambo G, Sullivan D, Mutambu SL, et al. High prevalence of molecular markers for resistance to chloroquine and pyrimethamine in Plasmodium falciparum from Zimbabwe. Parasitol Res 2007; 101(40): 1147-51.

[47] Mayxay M, Nair S, Sudimack D, et al. Combined molecular and clinical assessment of Plasmodium falciparum antimalarial drug resistance in the Lao People's Democratic Republic (Laos) Am J Trop Med Hyg 2007; 77(1): 36-43.

[48] Duah NO, Wilson MD, Ghansah A, et al. Mutations in Plasmodium falciparum chloroquine resistance transporter and multidrug resistance genes and treatment outcomes in Ghanaian children with uncomplicated malaria. J Trop Pediatr 2007; 53(1): 27-31. 